\title{
A novel 4 dimensional hyperchaotic system with its control, synchronization and implementation
}

\author{
Basil H. Jasim ${ }^{1}$, Anwer Hammadi Mjily², Anwer Mossa Jassim AL-Aaragee \\ ${ }^{1,3}$ Electrical Engineering Department, College of Engineering, University of Basrah, Iraq \\ ${ }^{2}$ Computer Center, University of Basrah, Iraq
}

\begin{tabular}{l}
\hline Article Info \\
\hline Article history: \\
Received Aug 27, 2020 \\
Revised Oct 8, 2020 \\
Accepted Dec 5, 2020 \\
\hline
\end{tabular}

Keywords:

Adaptive controller

Chaotic

Hyperchaotic

Lyapunov exponents

Synchronization

\begin{abstract}
This paper presents a new hyperchaotic system which shows some interesting features, the system is 4-dimensional with 4 nonlinearities. An extensive numerical analysis has showed that the system has some interesting features and strange behaviors. The numerical analysis includes studying the effect of system parameters and initial conditions. Some of the important properties of the system with parameter set, in which the system is hyperchaotic, such as Lyapunov exponents and Lyapunov dimension, dissipation and symmetry are found and discussed. In the next part of our work, a tracking controller for the proposed system is designed and then a synchronization control system for two identical systems is designed. The design procedure uses combination of a simple synergetic control with adaptive updating laws to identify the unknown parameters derived basing on Lyapunov theorem. Hardware implementation based on microcontroller unit (MCU) board is proposed and tested and used to experimentally validate the designed control and synchronization systems. As an application, the designed synchronization system is used as a secure analogue communication system. Using MATLAB, Simulation study for the control and synchronization systems is presented. The simulation and experimental study have been showed excellent results.
\end{abstract}

This is an open access article under the CC BY-SA license.

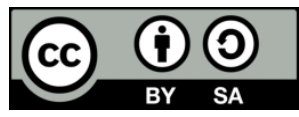

\section{Corresponding Author:}

Basil H. Jasim

Electrical Engineering Department

College of Engineering

University of Basrah, Iraq

Email: basil.jasim@uobasrah.edu.iq

\section{INTRODUCTION}

The dynamical system which highly effected by initial conditions is known as chaotic system. Chaotic systems are characterized by their strange attractors. Due to their attraction, theoretical importance and possible scientific and engineering applications, chaotic systems received great attention in the literature and scientific research [1]. Analytically, there are several methods to investigate the existence of chaotic behavior in dynamical systems, the most important of these methods are Lyapunov exponents and bifurcation diagrams [2]. Dynamical systems having positive maximum Lyapunov exponent are chaotic [3]. The largest maximum positive exponent system is the more chaos one. Hyperchaotic system is a chaotic system with more than one positive Lyapunov exponents $[4,5]$. Hyperchaotic systems show more dynamical complexity comparing with that systems which have one Lyapunov exponent or simply ordinary chaotic systems [6]. Chaotic systems get great attention in last decades due to their theoretical and practical applications and in various fields such as in secure communication, neural networks, laser, nonlinear circuits, mobile robots, oscillators, artificial neural networks, chemical reactors, finance systems, circuits and others [7-11]. 
According to some researchers, chaos can be regarded as the third largest scientific revolution in the past and current century $[12,13]$.

Since the first arise of chaotic systems by Lorenz [14], several chaotic and hyperchaotic systems with ordinary differential equations, complex and fractional order have been introduced and extensively studied [4, 7, 9, 15-17]. Despite of this fact "there was a lot of new chaotic and hyperchaotic systems proposed along the last years," but it is still advantageous for the field of chaotic in theoretical and practical areas to find and analyses new chaotic systems [17, 18]. Due to their dynamical complexity over chaotic systems, hyperchaotic systems admits more benefits in some applications, especially in the secure communication where it has been shown that encryption with hyperchaotic system gives more efficient secure communication system [19].

In classical chaotic theory, the excitation of strange attractors of chaotic systems comes from their unstable equilibria, so they are referred to as self-excited chaotic systems [8,9]. More recently, another type of chaotic systems has been appeared, where no equilibria or just a stable equilibrium point system with chaotic behavior [20,21]. These types of systems have been known as hidden attractors chaos and the reason for this name is very clear. Hidden attractor chaotic systems became now well known as the second source for chaotic systems [22]. Synchronization of two or more chaotic systems received considerable attention due to its theoretical and practical importance in several fields such as secure communication [23, 24]. Sliding mode control theory, adaptive control, active control and other approaches have been used for solving the challenges of the problem of chaotic systems synchronization [25].

Motivated by the previous discussion, we present in this work, a new hyperchaotic system with some interesting features. An extensive study including the important dynamical properties of the proposed system is presented. A tracking and synchronization controllers are designed. Hardware MCU based implementation is proposed and finally an application of the synchronization system is proposed and tested by simulation and experimental study.

The rest of the paper is organized as follows: In section 2, the new system is introduced with a brief detail. The dynamical system analysis and its properties are presented in section 3. A tracking control system design for the system is presented in section 4 . In section 5, a synchronization control system is designed. In section 6, a description of the proposed hardware implementation is given. In section 7, simulation and experimental study is presented and used to validate the effectiveness of the control and synchronization system and to use the designed synchronization system to build and test a secure communication system. Finally, in section 7, paper conclusion has been drawn.

\section{THE PROPOSED HYPERCHAOTIC SYSTEM}

The following system of equations which have three quadratic and one quartic nonlinearity terms represents the new system:

$$
\begin{aligned}
& \dot{x}_{1}=a x_{2}-12 x_{1} \\
& \dot{x}_{2}=b x_{1} x_{4} \\
& \dot{x}_{3}=10 x_{1}^{2}-12 x_{3}^{2} \\
& \dot{x}_{4}=20-0.5 x_{2}^{4}
\end{aligned}
$$

In this system, $\mathrm{a}$ and $\mathrm{b}$ are positive real parameters.

System (1) shows chaotic behavior for a certain value of parameters set and for certain initial conditions. The system characterized by its high independent on initial conditions. It has been found that for a certain set of parameters, the system may exhibit chaotic, hyperchaotic, periodic and quasi periodic motions depending on the initial conditions.

\section{SYSTEM ANALYSIS}

In this section, the system is analyzed. The analysis will mainly base on numerical simulation. Equilibria analysis, Phase portrait of the system, bifurcation diagrams and Lyapunov exponents are the main tools used in analyzing the system.

\subsection{Equilibrium points}

The equilibrium points play significant role in the dynamical behavior of the nonlinear systems. Finding these points and investigating their types is essential in analyzing nonlinear systems. The only restriction in investigating systems stability of nonlinear systems by linearization technique is the case of zero real part of the Eigen values of one or more of the equilibria points. To obtain the equilibrium points of a 
system, the left side of the system equations should be equated to zero. Then, solving for the states of the system (the $X=\left\{\mathrm{x}_{1}, \mathrm{x}_{2}, \mathrm{x}_{3}, \mathrm{X}_{4}\right\}$ vector). The equilibrium points have been found as a function of system parameters. The system has 4 equilibria and as listed:

$$
\begin{aligned}
& x_{1}=\left\{a(2 f)^{\frac{1}{4}},(2 f)^{\frac{1}{4}}, \frac{a(2 f)^{\frac{1}{4}}}{12}, 0\right\} \\
& x_{2}=\left\{-a(2 f)^{\frac{1}{4}},-(2 f)^{\frac{1}{4}}, \frac{a(2 f)^{\frac{1}{4}}}{12}, 0\right\} \\
& x_{3}=\left\{a(2 f)^{\frac{1}{4}},(2 f)^{\frac{1}{4}},-\frac{a(2 f)^{\frac{1}{4}}}{12}, 0\right\} \\
& x_{1}=\left\{-a(2 f)^{\frac{1}{4}},-(2 f)^{\frac{1}{4}},-\frac{a(2 f)^{\frac{1}{4}}}{12}, 0\right\}
\end{aligned}
$$

where $\left(C_{N \square}\right)_{c o n e}$ is the normal-force-curve slope of pointed cone with $\beta / \lambda_{N}^{\prime}$ and $\eta_{C y l}^{\prime}$, and $\left(C_{N \square}\right)_{s p h e r e}$ is the normal-force-curve slope of hemispherical nose. listed in (3).

To determine the types of these points, the spectral or Eigen values of these points are found and as

$$
\begin{aligned}
& e g\left(x_{1}\right)=\left\{-12,0,-2 a(2 f)^{\frac{1}{4}},-2(2 f)^{\frac{3}{4}}\right\} \\
& e g\left(x_{2}\right)=\left\{-12,0,-2 a(2 f)^{\frac{1}{4}}, 2(2 f)^{\frac{3}{4}}\right\} \\
& \operatorname{eg}\left(x_{1}\right)=\left\{-12,0,2 a(2 f)^{\frac{1}{4}},-2(2 f)^{\frac{3}{4}}\right\} \\
& e g\left(x_{1}\right)=\left\{-12,0,2 a(2 f)^{\frac{1}{4}}, 2(2 f)^{\frac{3}{4}}\right\}
\end{aligned}
$$

Then, all of the Eigen values of the fixed points have zero value, which means that these points are non-hyperbolic. Then, linearization cannot be useful or enough tool to determine the stability and behavior of our system in neighborhoods of the equilibrium points and the overall system stability. The non-hyperbolic points are center points but their stability cannot be ensured by linearization.

To get some indication about the stability of the equilibrium points, the system has been simulated using initial conditions which are close to the equilibrium points. Choosing $a, b=\{17,20\}$, getting then the equilibrium points of the system at these parameters. The numerical simulation shows that the system admits periodic motion when simulated with initial conditions close to $\mathrm{x}_{1}$ and $\mathrm{x}_{2}$, while it is unstable when using close initial conditions to $\mathrm{x}_{3}$ and $\mathrm{x}_{4}$, where $\mathrm{x}_{1}, \mathrm{x}_{2}, \mathrm{x}_{3}$ and $\mathrm{x}_{4}$ are the first to fourth equilibria points. This shows that the system may exhibit chaos behavior with self-excitation or by hidden attractors.

\subsection{Effects of the system parameters}

To study the effect of the system parameters on the system behavior, bifurcation diagrams have been used. First, the parameter $b$ has been fixed to 7 and a has been changed from 0.1 to 20 with 0.01 as a step size. The system was simulated at each step for 500 second with $\{1,1,1,1\}$ as an initial condition, the maxima of $\mathrm{x}_{2}$ have been found. We ignored the first 100 second of flow to ensure destroying transient flow. Figure 1 shows the resultant diagram. Then, the same process has been repeated for $b$ with $a=7$, Figure 2 shows the bifurcation diagram in this case.

It is known from literature that there are three main routes to chaos. The first one is period doubling in which the period of periodic motion of the system is doubled at specific point of bifurcation parameter and then and at a specific value of bifurcation parameter the motion becomes chaotic. The second route is the intermittency chaos in which periodic motion is replaced by chaotic at a certain value of bifurcation parameter. The last route is quasi periodic to chaos in which the system motion changes from fixed to periodic and quasi periodic and lastly chaotic motion appear. Analyzing Figures 1 and 2, shows that the system rout to chaos is the intermittency chaos. From this figures, it can also be noted that periodic motion appears interchangeably with chaos motion in some region of bifurcation diagram. 


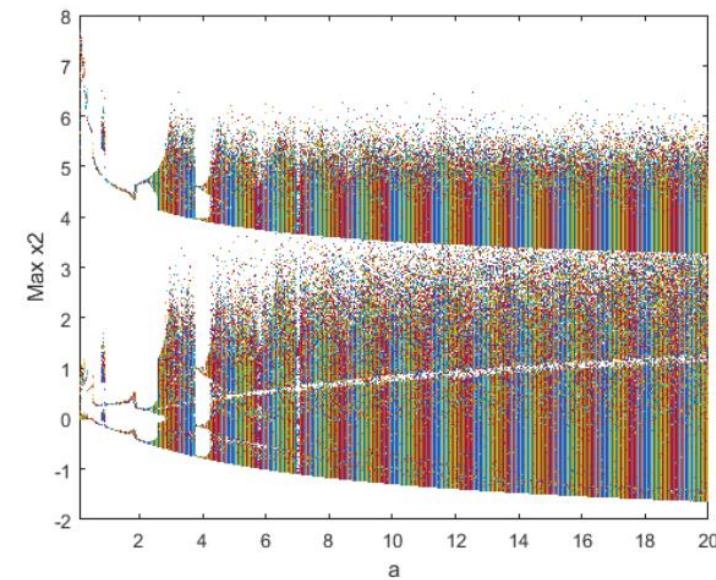

Figure 1. Bifurcation diagram for a parameter

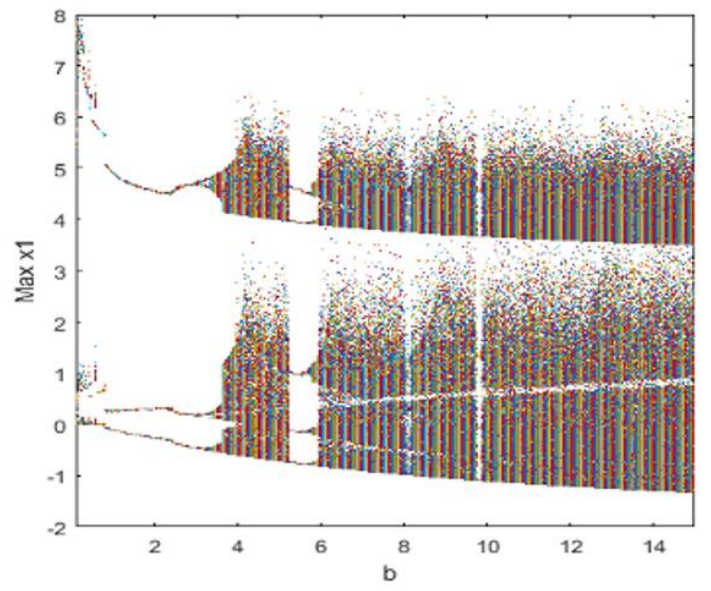

Figure 2. Bifurcation diagram for $b$ parameter

From the bifurcation diagrams, the regions in which the system is chaotic is clear and from these regions we selected $a=17$ and $b=20$. In the rest of this paper the system parameters will be fixed at these values. At these values of system parameters, the system is hyperchaotic. To prove the hyperchaotic nature of the system, we used Lyapunov exponents. The Lyapunov exponents have been found by using Alan wolf algorithm [26]. Figure 3 shows the dynamics of Lyapunov exponents for these values of $a$ and $b$ and for 1000 seconds. The settling values of the exponents are:

$$
L_{1}=2.56, L_{2}=0.023, L_{3}=-14.6, L_{4}=-40.24
$$

Then, the first and the second exponents are positive which means that the system is hyperchaotic. Figure 4 shows the 2 dimensional hyperchaotic attractor of the system for $\mathrm{x}_{1}, \mathrm{x}_{2}$ phase plane and Figure 5 shows phase portrait of the system for $\mathrm{x}_{1}, \mathrm{x}_{3}$ plane.

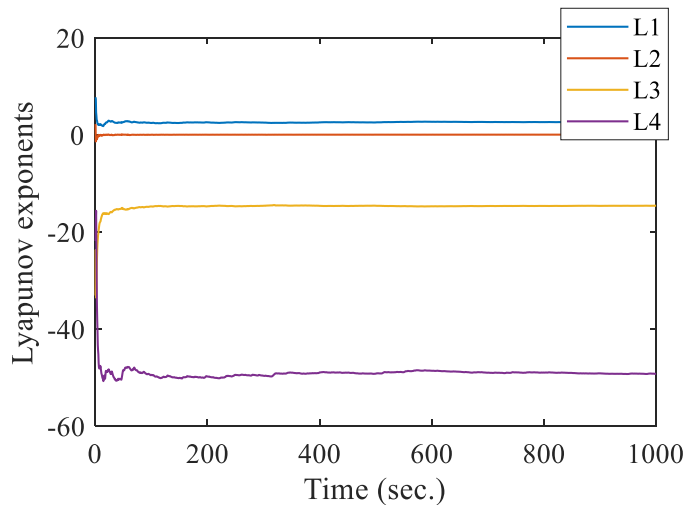

Figure 3. Lyapunov exponents

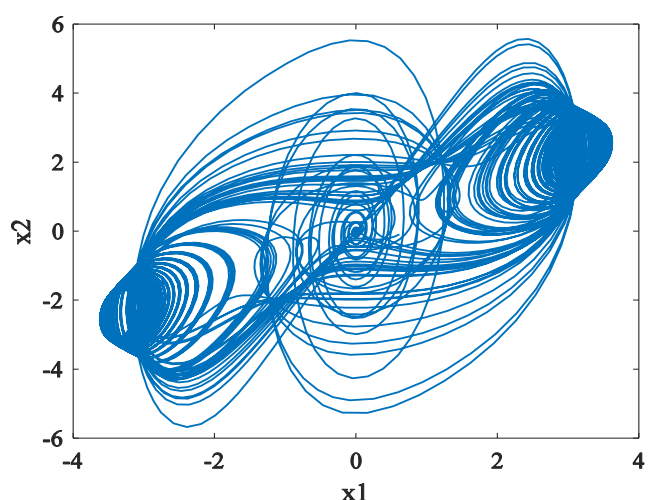

Figure 4. 2 D phase portrait of $\mathrm{x}_{1}, \mathrm{x}$

\subsection{The effects of the initial conditions}

In this subsection, the initial condition effects on the system behavior are studied. As mentioned previously, the system parameters are fixed at $a=17$ and $b=20$.

\subsubsection{The sensitivity to the initial conditions}

The system shows very high sensitivity to small changes in initial condition. The high sensitivity to initial condition of the system is tested by simulating the system for initial condition $X 0=\{1,1,1,1\}$ and for 100 seconds, then a small perturbation to this initial condition is entered where X0 becomes $\{1.00001,1,1,1\}$ and the system has been simulated again for the same period of time. Figure 6 shows the motion of $\mathrm{x}_{1}$ from 10 to 20 seconds. It is clear that the system motion has changed significantly for this small change of IC. 


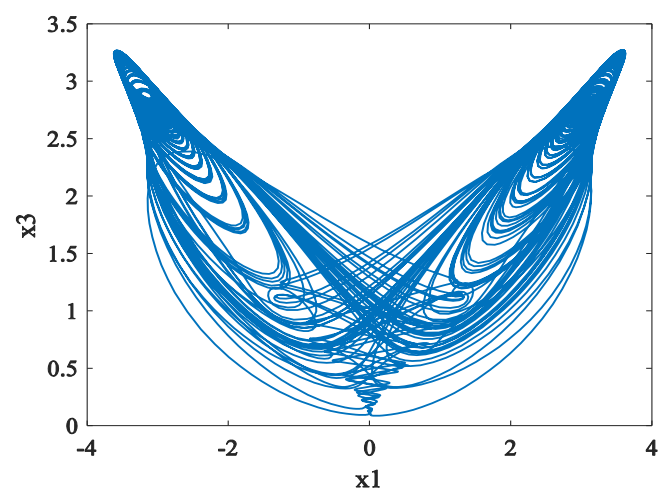

Figure 5. 2 D phase portrait of $\mathrm{x}_{1}, \mathrm{x}_{3}$

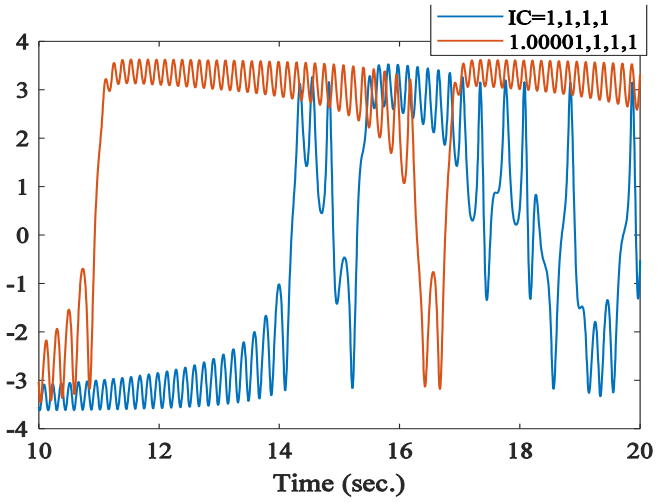

Figure 6. The response of $\mathrm{x}_{1}$ for 2 close ICs

\subsubsection{The effects of the initial conditions on the system behavior}

For the selected parameter values, the system is hyperchaotic for most initial conditions as shown previously. Even though the system shows different behavior for some initial conditions. It shows periodic and quasi periodic motions for some ICs. Also, it shows coexisting limit cycle attractors for some initial conditions. It also shows a strange behavior for some initial conditions, where the system motion is settle down to a periodic motion for a long time (more than 400 second), but after that this motion is replaced by a quasi-periodic motion. Then, the system for these ICs shows 2 transient motion, the first is fast and destroyed after a few seconds and the second which is a periodic motion takes a long time and the settling motion is quasi periodic. Table 1 lists some examples of these initial condition's effects and Figure 7 to 9 show the corresponding responses.

Table 1. The ICs and the corresponding motion description

\begin{tabular}{ll}
\hline \multicolumn{1}{c}{ The initial conditions } & \multicolumn{1}{c}{ The behavior } \\
\hline$\{1.1,2.5,2.1,0\},\{-1.1,-2.5,2.1,0\}$ & 2 coexisting limit cycle \\
$\{1.1,2.5,2.1,0\},\{1.11,2.51,2.11,0.01\},\{1.12,2.52,2.12,0.02\},\{1.12,2.52,2.12,0.02\}$ & 4 coexisting limit cycle \\
$\{13.4,1,1,1\}$ & Transient, periodic, quasi periodic \\
\hline
\end{tabular}

\subsection{Some dynamical features} discussed.

In this subsection, some important dynamical properties of the proposed system are found and

\subsubsection{Symmetry}

It can easily be noted that the system is invariant under the transformation:

$$
\left(x_{1}, x_{2}, x_{3}, x_{4}\right) \rightarrow\left(-x_{1},-x_{2}, x_{3}, x_{4}\right)
$$

which means that if $x_{1}, x_{3}, x_{1}, x_{3}$ are a solution for the system equations, then $-x_{1},-x_{3}, x_{1}, x_{3}$ is also a solution for it,

\subsubsection{Lyapunov dimension}

The Lyapunov dimension is a measure of system chaotic behavior degree, it can be defined according to Kaplan-York [27] conjecture and as (5):

$$
D_{k}=J+\frac{\sum_{i=1}^{J} L_{i}}{\left|L_{J+1}\right|}
$$

where J should be selected such that $\sum_{i=1}^{J} L_{i}>0$ and $\sum_{i=1}^{J+1} L_{i}<0$.

Then, for our system:

$$
D_{k}=3+\frac{\sum_{i=1}^{3} L_{i}}{\left|L_{4}\right|}=3+\frac{L_{1}+L_{2}+L_{3}}{\left|L_{4}\right|}=2.76
$$



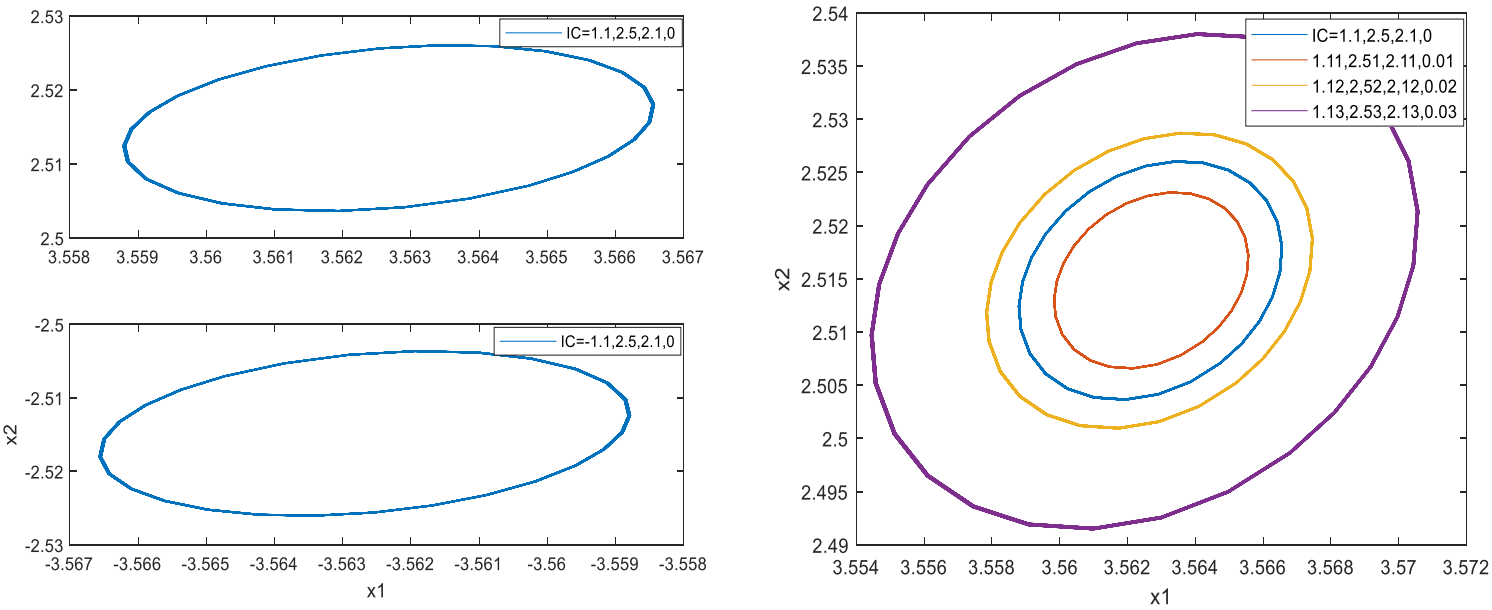

Figure 7. 2 coexisting limit cycles attractors

Figure 8.4 coexisting limit cycles attractors

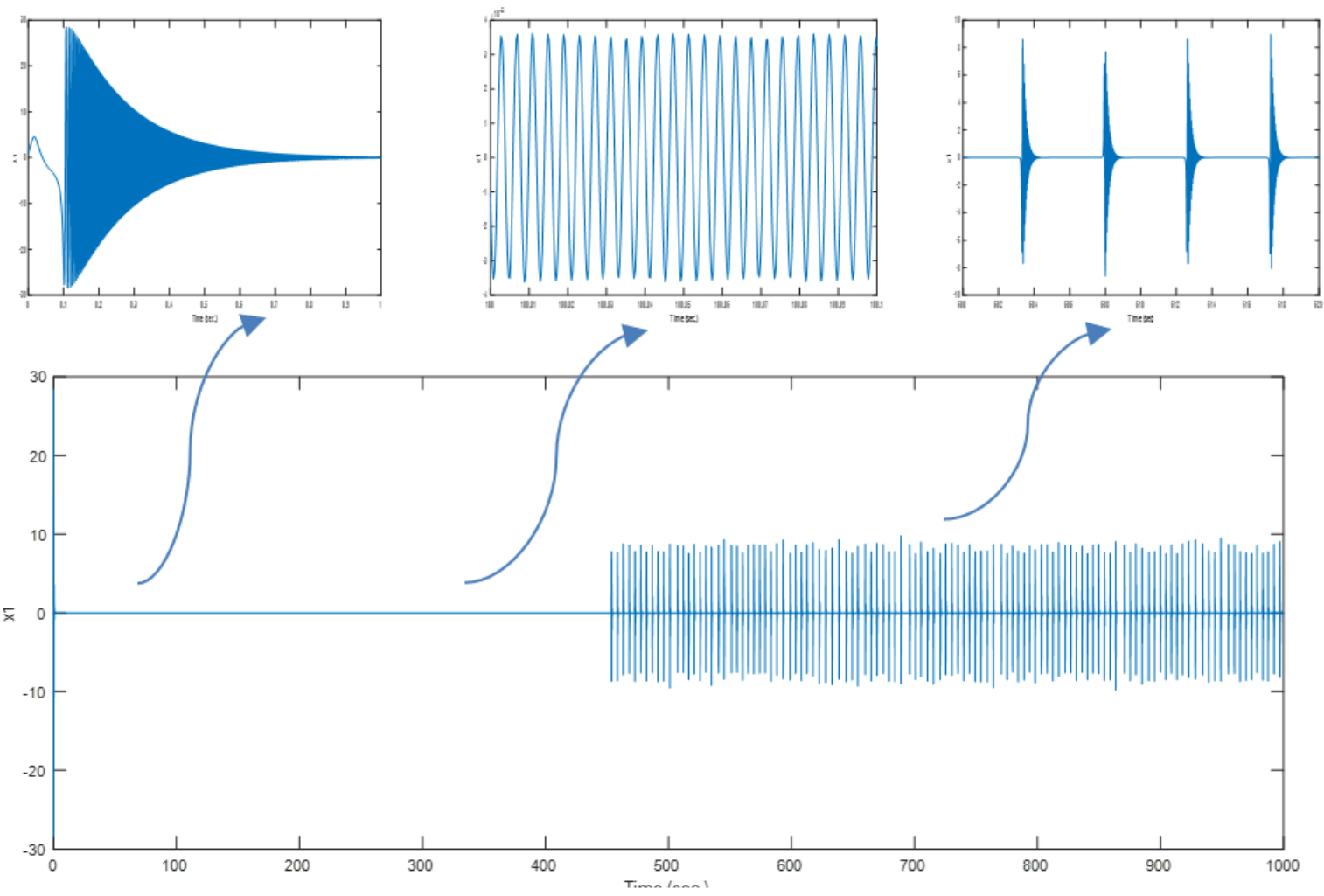

Figure 9. Response of system for IC $=\{13.4,1,1,1\}$

\section{TRACKING CONTROL DESIGN}

A controller to track a specific reference signals for the states of the system is designed in this section. To this end, we suppose that a vector of control input signals $U=\left\{u_{1}, u_{2}, u_{3}, u_{4}\right\}$ is added to the system, then the system equations now read:

$$
\begin{aligned}
& \dot{x}_{1}=a x_{2}-12 x_{1}+u_{1} \\
& \dot{x}_{2}=c x_{1} x_{3}+u_{2} \\
& \dot{x}_{3}=10 x_{1}^{2}-12 x_{3}^{2}+u_{3} \\
& \dot{x}_{4}=20-0.5 x_{2}^{4}+u_{4}
\end{aligned}
$$


In the controller design, we assumed that the system parameters a and b are unknown, so they need to be estimated. Simple synergetic control design with adaptive laws for unknown parameters estimation are used to design the controller. The design procedure relay on selecting the control inputs such that the following error dynamics of the system is satisfied:

$$
\dot{e}_{l}+k_{i} e_{i}=0, \quad \mathrm{i}=1,2,3,4
$$

In this equation, $e_{i}=r_{i}-x_{i}, r_{i}$ is the desired output of the system states $x_{i}$ and $k_{i}$ are design parameters which are strictly positive parameters for stable system and their values should be selected such that we get the desired response for error system. Substituting (6) into (7) and solving for U, the following is obtained:

$$
\begin{aligned}
& u 1=\dot{r}_{1}+k_{1} e_{1}-a_{n} x_{2}+12 x_{1} \\
& u 2=\dot{r}_{2}+k_{2} e_{2}-b_{n} x_{1} x_{3} \\
& u 3=\dot{r}_{3}+k_{3} e_{3}-10 x_{1}^{2}+12 x_{3}^{2} \\
& u 4=\dot{r}_{4}+k_{4} e_{4}-20+0.5 x_{2}^{4}
\end{aligned}
$$

where, $a_{n}$ and $b_{n}$ are the estimates of a and b. Substituting (8) into (6), the controlled system is obtained:

$$
\begin{aligned}
& \dot{x}_{1}=\dot{r}_{1}+k_{1} e_{1}+\left(a-a_{n}\right) x_{2} \\
& \dot{x}_{2}=\dot{r}_{2}+k_{2} e_{2}+\left(b-b_{n}\right) x_{1} x_{3} \\
& \dot{x}_{3}=\dot{r}_{3}+k_{3} e_{3} \\
& \dot{x}_{4}=\dot{r}_{4}+k_{4} e_{4}
\end{aligned}
$$
obtained:

using $x_{i}=r_{i}-e_{i}$ and $\dot{x}_{\imath}=\dot{r}_{l}-\dot{e}_{\iota}$ in 9 and after some manipulation, the following error dynamics is

$$
\begin{aligned}
& \dot{e}_{1}=a_{e} e_{2}-k_{1} e_{1}-a_{e} r_{2} \\
& \dot{e}_{2}=-k_{2} e_{2}+b_{e}\left(r_{1} e_{3}+e_{1} r_{3}-r_{1} r_{3}-e_{1} e_{3}\right) \\
& \dot{e}_{3}=-k_{3} e_{3} \\
& \dot{e}_{4}=-k_{4} e_{4}
\end{aligned}
$$

In $10, a_{e}$ and $b_{e}$ are the errors between real and estimated values of a and b, i.e. $a_{e}=a-a_{n}$ and $b_{e}=b-b_{n}$. Now, to stabilize the dynamics in (10), the estimated values should converge to the unknown parameter values, a Lyapunov theory is used to find update laws for $a_{n}$ and $b_{n}$ to converge to a and $\mathrm{b}$ and obtaining stable dynamics. The following positive definite function is selected as a Lyapunov function candidate:

$$
V(t)=1 / 2\left(e_{1}^{2}+e_{2}^{2}+e_{3}^{2}+e_{4}^{2}+a_{e}^{2}+b_{e}^{2}\right)
$$

Differentiating $\mathrm{V}(\mathrm{t})$ :

$$
\dot{V}(t)=e_{1} \dot{e_{1}}+e_{2} \dot{e_{2}}+e_{3} \dot{e_{3}}+e_{4} \dot{e_{4}}-\dot{a}_{n} a_{e}-\dot{b}_{n} b_{e}
$$

Substituting $\dot{e}_{\iota}$ from 10 and rearranging, the following result is obtained:

$$
\begin{aligned}
\dot{V}(t)=-k_{1} e_{1}- & k_{2} e_{2}-k_{3} e_{3}-k_{4} e_{4}+a_{e}\left(e_{1} e_{2}-r_{2} e_{1}-\dot{a}_{n}\right) \\
& +b_{e}\left(r_{1} e_{2} e_{3}+r_{3} e_{1} e_{2}-e_{2} r_{1} r_{3}-e_{1} e_{2} e_{3}-\dot{b}_{n}\right)
\end{aligned}
$$

If we update $a_{n}$ and $b_{n}$ as in (14):

$$
\begin{aligned}
& \dot{a}_{n}=e_{1} e_{2}-r_{2} e_{1} \\
& \dot{b}_{n}=r_{1} e_{2} e_{3}+r_{3} e_{1} e_{2}-e_{2} r_{1} r_{3}-e_{1} e_{2} e_{3}
\end{aligned}
$$

Then

$$
\dot{V}(t)=-k_{1} e_{1}-k_{2} e_{2}-k_{3} e_{3}-k_{4} e_{4}
$$

And this is a semidefinite function, ensuring then system stability. 


\section{SYNCHRONIZATION CONTROLLER DESIGN}

In this section, a controller to synchronize two identical of the proposed system is designed. The system (1) is uncontrolled system or the master and its states output should be tracked or synchronized by the second system which is often called the slave in the literature. The Slave system is the same system (1) added to it the control signals $U=\left\{u_{1}, u_{2}, u_{3}, u_{4}\right\}$. Master system is represented by system 1 with a and $\mathrm{b}$ assumed to be unknown, while the slave system is represented by the following system:

$$
\begin{aligned}
& \dot{y}_{1}=a y_{2}-12 y_{1}+u_{1} \\
& \dot{y}_{2}=c y_{1} y_{3}+u_{2} \\
& \dot{y}_{2}=10 y_{1}^{2}-12 y_{3}^{2}+u_{3} \\
& \dot{y}_{4}=20-0.5 y_{2}^{4}+u_{4}
\end{aligned}
$$

The synchronization error is defined as:

$$
e_{i}=y_{i}-x_{i}, i=1,2,3,4
$$

using this relation with (1), (16) and $\dot{e}_{\iota}=\dot{y}_{l}-\dot{x}_{\imath}, i=1,2,3,4$, the following system of equations is obtained:

$$
\begin{aligned}
& \dot{e}_{1}=a e_{2}-12 e_{1}+u_{1} \\
& \dot{e}_{2}=b\left(y_{1} y_{4}-x_{1} x_{4}\right)+u_{2} \\
& \dot{e}_{3}=10\left(y_{1}^{2}-x_{1}^{2}\right)-12\left(y_{3}^{2}-x_{3}^{2}\right)+u_{3} \\
& \dot{e}_{4}=0.5\left(x_{2}^{4}-y_{2}^{4}\right)+u_{4}
\end{aligned}
$$

Using similar design procedure used in tracking controller design, our target here also is to satisfy the following error dynamics:

$$
\dot{e}_{\imath}+k_{i} e_{i}=0, \quad \mathrm{i}=1,2,3,4
$$

Combining 19 and 18 and solving for U, we obtain:

$$
\begin{aligned}
& u 1=-k_{1} e_{1}-a_{n} e_{2}+12 e_{1} \\
& u 2=-k_{2} e_{2}-b_{n}\left(y_{1} y_{4}-x_{1} x_{4}\right) \\
& u 3=-k_{3} e_{3}-10\left(y_{1}^{2}-x_{1}^{2}\right)+12\left(y_{3}^{2}-x_{3}^{2}\right) \\
& u 4=-k_{4} e_{4}-0.5\left(x_{2}^{4}-y_{2}^{4}\right)
\end{aligned}
$$

where $a_{n}$ and $b_{n}$ are the estimates of a and $\mathrm{b}$.

Substituting (20) into 18, yields:

$$
\begin{aligned}
& \dot{e}_{1}=-k_{1} e_{1}+a_{e} e_{2} \\
& \dot{e}_{2}=-k_{2} e_{2}+b_{e}\left(y_{1} y_{4}-x_{1} x_{4}\right) \\
& \dot{e}_{3}=-k_{3} e_{3} \\
& \dot{e}_{4}=-k_{4} e_{4}
\end{aligned}
$$

where $a_{e}$ and $b_{e}$ are the error between the real parameter values of a and b and the estimate values $a_{n}$ and $b_{n}$.

The parameter estimation will be designed by Lyapunov theory. The following function is selected as a Lyapunov function candidate:

$$
V(t)=1 / 2\left(e_{1}^{2}+e_{2}^{2}+e_{3}^{2}+e_{4}^{2}+a_{e}^{2}+b_{e}^{2}\right)
$$

then,

$$
\dot{V}(t)=e_{1} \dot{e_{1}}+e_{2} \dot{e_{2}}+e_{3} \dot{e_{3}}+e_{4} \dot{e_{4}}-\dot{a}_{n} a_{e}-\dot{b}_{n} b_{e}
$$

and,

$$
\dot{V}(t)=-k_{1} e_{1}-k_{2} e_{2}-k_{3} e_{3}-k_{4} e_{4}+a_{e}\left(e_{1} e_{2}-\dot{a}_{n}\right)-b_{e}\left(e_{2}\left(y_{1} y_{3}-x_{1} x_{3}\right)+\dot{b}_{n}\right)
$$

If we put: 


$$
\begin{aligned}
& \dot{a}_{n}=e_{1} e_{2} \\
& \dot{b}_{n}=e_{2}\left(y_{1} y_{4}-x_{1} x_{4}\right)
\end{aligned}
$$

then,

$$
\dot{V}(t)=-k_{1} e_{1}-k_{2} e_{2}-k_{3} e_{3}-k_{4} e_{4}
$$

which is a negative semidefinite and the prove of system stability is completed.

\subsection{System implementation}

The proposed system has been implemented using microcontroller platform. We propose implementing of the system using the so-called Arduino MCU board, where Arduino uno board has been used to implement the system. Arduino boards are easy to use MCUs development boards. The used board is the Arduino uno which has $32 \mathrm{~KB}$ flash memory for storing the program and $2 \mathrm{~KB}$ of SRAM memory for storing program data and $1 \mathrm{~KB}$ EEPOM which can be used for storing static data of the program. These specifications can cover all the requirements of implementing our system. The implementation program uses fourth order Runge-Kuta method to solve the ODE system of equations of our system with step size equal to 0.001 second. For the purpose of testing the system, we have used MATLAB to plot the phase portraits of the system states plane where the MCU board and laptop have been connected via serial communication. Appendix A shows the implementation program and appendix B shows the program used to receive the data from MCU board. Figure 10 shows the phase portrait of $\mathrm{x}_{1}, \mathrm{x}_{2}$ plane received from MCU board for 30 seconds run time.

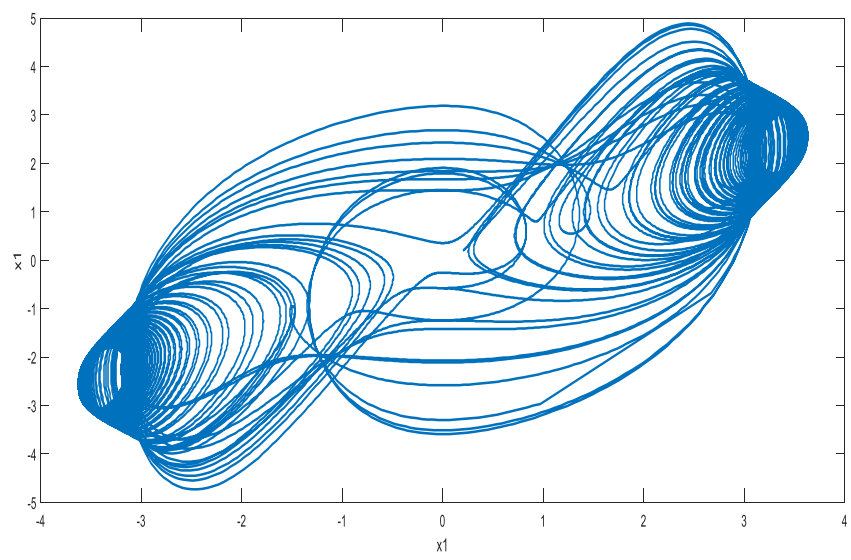

Figure 10. $\mathrm{x}_{1}, \mathrm{x}_{2}$ portrait of the implemented system

\section{SIMULATION AND EXPERIMENTAL STUDY}

In this section, the theoretical results of tracking control system and synchronization of the proposed hyperchaotic system are investigated by simulation and experimentally. Also, a secure communication using our synchronization system is introduced and tested. As mentioned earlier, MATLAB 2018a script is used to write the simulation programs. The experimental study relayed on the system implemented on the MCU Arduino board and as described in the previous section.

\subsection{Control system}

The tracking control system has been tested by simulation with two vectors of reference signals. First, we assumed that the system is to be stabilized from initial conditions, then, Reference signals $=R=$ $\left\{r_{1}, r_{2}, r_{3}, r_{4}\right\}=\{0,0,0,0\}$ The initial conditions for the states $X(0)=\left\{x_{1}(0), x_{2}(0), x_{3}(0), x_{4}(0)\right\}=$ $\{1,-1,3,2\}$. The states responses of the system are shown in Figure 11. Next, sinusoidal signals are assumed as a reference signal. Using the same initial conditions, the responses are shown in Figure 12. From Figure 11 and 12 , it is very clear that the designed control system performance is excellent.

$$
R=\left\{r_{1}, r_{2}, r_{3}, r_{4}\right\}=\{\sin (20 t), 2 \sin (20 t), 3 \sin (20 t), 4 \sin (20 t),\}
$$




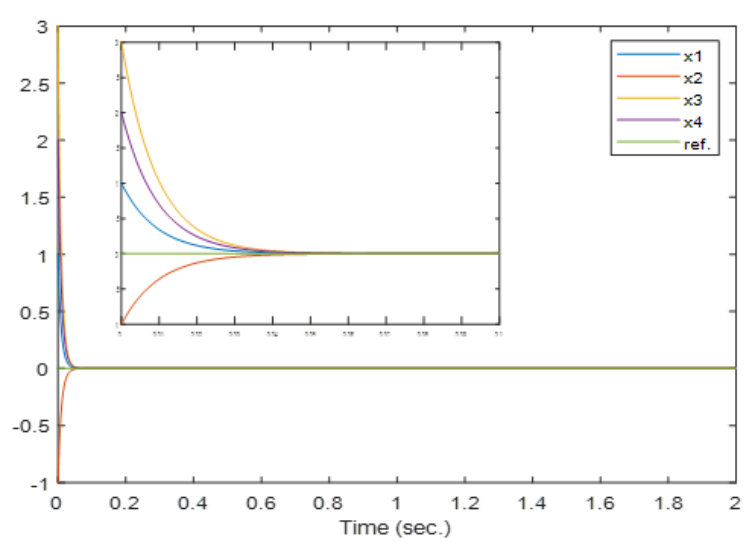

Figure 11. System stabilization from initial conditions

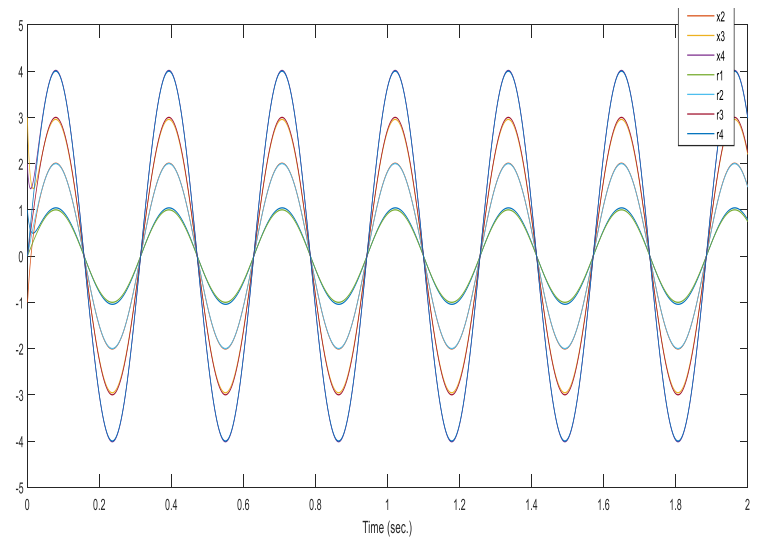

Figure 12. Tracking system responses for sinusoidal signals

\subsection{Synchronization system}

The designed synchronization system is tested by using the MCU based system designed in the previous section as a master and the slave system is implemented on computer side using MATLAB with the following initial conditions for master and slave respectively. The synchronization errors are shown in Figure 13. This figure shows an excellent synchronization error response.

$$
\begin{aligned}
& X=\left\{x_{1}, x_{2}, x_{3}, x_{4}\right\}=\{1,2,3,1\} \\
& Y=\left\{y_{1}, y_{2}, y_{3}, y_{4}\right\}=\{2,1,1,2\}
\end{aligned}
$$

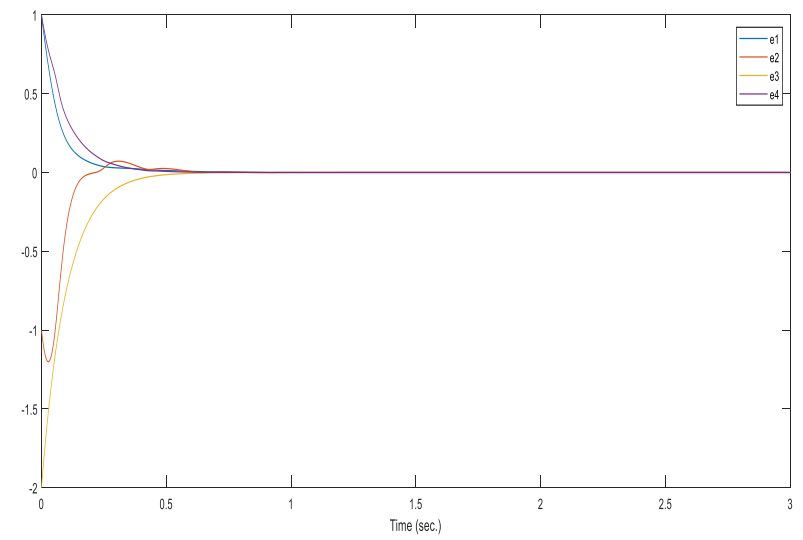

Figure 13. Errors of the synchronization system

\subsection{Secure communication application}

In this subsection, an application of chaotic system synchronization is presented. In analog secure communication using synchronized chaotic systems, the transmitted signal (sig) is added to one of the master systems states ( say $x_{1}$ ) to form the scripted signal (tr) to be transmitted to the receiver side with other signal required by slave to synchronize its output with master system. At the receiver side, the scripted (tr) signal is subtracted from the counterpart state of the slave ( $y_{l}$ in our case) to form the decrypted signal (rs), then: At the transmitter:

$$
\operatorname{tr}=\operatorname{sig}+x_{1}
$$

And at the receiver:

$$
r s=t r-y_{1}
$$


It is clear that if full synchronization is achieved, signal is completely retrieved, i.e. $r s=$ sig.

In our test, we used the implemented system on MCU based as a transmitter side and the laptop as a receiver side. A sinusoidal signal $(\operatorname{sig}=4 \sin (30 t))$ is selected as a signal to be transmuted. Figure 14 shows the transmitted (sig) signal and the received (rs) signal. From this figure, it can be noted that the transmitted signal is tracked at the receiver with no error and in very short time.

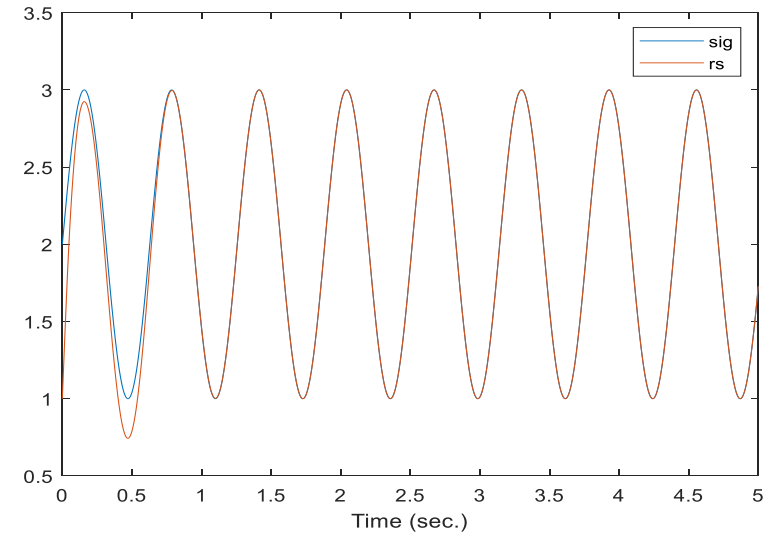

Figure 14. The transmitted and received signals and error

\section{CONCLUSION}

In this paper, a new 4-dimensional hyperchaotic system has been proposed. The system has been extensively analyzed and its dynamical properties have been investigated. It has been found that the system has some interesting features and strange behaviors. After that, a tracking and synchronization systems for the introduced system have been designed, assuming unknown system parameters. The design procedure depended on simple controller with Lyapunov theory for the purpose of deriving update lows for the unknown parameters. Hardware realization is proposed where MCU board is used to implement the system. It is well known that MCU implementation gives a lot of features like cost effective, reliability and flexibility. Simulation and experimental study have showed the effectiveness of the designed control and synchronization systems and also the effectiveness of the secure communication system which can be designed basing on the designed synchronization system.

\section{REFERENCES}

[1] A. A. Oumate, S. Vaidyanathan, K. B. Gambo, and B. A. Mohamadou, "Analysis, Circuit Design and Synchronization of a New Hyperchaotic System with Three Quadratic Nonlinearities," Nonlinear Dynamical Systems with Self-Excited and Hidden Attractors, pp. 251-270, 2018, doi: 10.1007/978-3-319-71243-7_11.

[2] E. Mahmoud and F. Abood, "A novel sort of adaptive complex synchronizations of two indistinguishable chaotic complex nonlinear models with uncertain parameters and its applications in secure communications," Results in Physics, vol. 7, pp. 4174-4182, 2017, doi: 10.1016/j.rinp.2017.07.050.

[3] Y. Liu, Q. Yang, and G. Pang, "A hyperchaotic system from the Rabinovich system," J. Computational Applied Mathematics, vol. 234, pp. 101-113, 2010, doi: 10.1016/j.cam.2009.12.008.

[4] E. Mahmoud, "Generation and suppression of a new hyperchaotic nonlinear model with complex variables," Applied Mathematical Modelling, vol. 38, no. 17-18, pp. 4445-4459, 2014, doi: 10.1016/j.apm.2014.02.025.

[5] A. Y. Aguilar-Bustos and C. Cruz-Hernández, "Synchronization of discrete-time hyperchaotic systems: An application in communications," Chaos, Solitons and Fractals, vol. 41, no. 3, pp. 1301-1310, 2009, doi: 10.1016/j.chaos.2008.05.012.

[6] T. Sangpet and S. Kuntanapreeda, "Finite-time synchronization of hyperchaotic systems based on feedback passivation," Chaos, Solitons and Fractals, vol. 132, 2020.

[7] A. Yousefpour, H. Jahanshahi, J. M Munoz-Pacheco, S. Bekiros, and Z. Wei, "A fractional-order hyper-chaotic economic system with transient chaos," Chaos Solitons \& Fractals, vol. 130, 2019, doi: 10.1016/j.chaos.2019.109400.

[8] Ph. Faradja and G. Qi, "Analysis of multistability, hidden chaos and transient chaos in brushless DC motor," Chaos Solitons \& Fractals, vol. 132, 2020, doi: 10.1016/j.chaos.2020.109606.

[9] Y. Cao, "Chaotic synchronization based on fractional order calculus financial system," Chaos, Solitons \& Fractals, vol. 130, 2020, doi: 109410. 10.1016/j.chaos.2019.109410. 
[10] S. Khorashadizadeh and M. Majidi, "Chaos synchronization using the Fourier series expansion with application to secure communications," AEU-International Journal of Electronics and Communications, vol. 82, pp. 37-44, 2017, doi: 10.1016/j.aeue.2017.07.032.

[11] G. Al-Suhail, F. Tahir, M. Abd, V. T. Pham, and L. Fortuna, "Modelling of Long-Wave Chaotic Radar System for Anti-Stealth Applications," Communications in Nonlinear Science and Numerical Simulation, vol. 57, pp. 80-96, 2017, doi: 10.1016/j.cnsns.2017.09.008.

[12] J. Gleick, "Chaos: making a new science," Open Road Media, 2011.

[13] M. Dutta, K. Binoy, and A. Roy, "New fractional-order system displaying coexisting multiwing attractors; its synchronisation and circuit simulation," Chaos Solitons \& Fractals, vol. 130, 2020, doi: 109414. 10.1016/j.chaos.2019.109414.

[14] E. N Lorenz, "Deterministic nonperiodic flow," J. Atmos. Phys, vol. 20, no. 2, pp. 131-141, 1963, doi: 10.1175/1520-0469(1963)020\%3C0130:DNF\%3E2.0.CO;2.

[15] E. Mahmoud, "Dynamics and synchronization of new hyperchaotic complex Lorenz system," Mathematical and Computer Modelling, vol. 55, no. 7-8, pp. 1951-1962, 2012, doi: 10.1016/j.mcm.2011.11.053.

[16] M. Mossa Al-sawalaha, "Synchronization of different order fractional order chaotic systems using modify adaptive sliding mode control," Advances in difference equations, vol. 2020, no. 1, pp. 1-17, 2010, doi: 10.1186/s13662020-02876-7.

[17] M. Ababneh, "A new four-dimensional chaotic attractor," Ain Shams Engineering Journal, vol. 9, no. 4, pp. 1849-1854, 2017, doi: 10.1016/j.asej.2016.08.020.

[18] N. Averbeck and B. Raines, "Distributional chaos in dendritic and circular Julia sets," J. Math. Anal. Appl., vol. 428, no. 2, pp. 951-958, 2015.

[19] G. Alvarez, F. Montoya, M. Romera, and G. Pastor, "Breaking parameter modulated chaotic secure communication system," Chaos Solitons \& Fractals, vol. 21, no. 4, pp. 783-787, 2004.

[20] G. A. Leonov, N. V. Kuznetsov, and V. Vagaitsev, "Hidden attractor in smooth Chua sys- tems," Physica D, vol. 241, no. 18, pp. 1482-1486, 2012.

[21] M. Wang, X. Liao, Y. Deng, L. Zhijun, Y. Su, Z. Yongxin, and Y. Zeng, "Dynamics, synchronization and circuit implementation of a simple fractional-order chaotic system with hidden attractors," Chaos, Solitons \& Fractals, vol. 130, 2020, doi: 10.1016/j.chaos.2019.109406.

[22] S. Vaidyanathan, V.T. Pham, C. Volos and A. Sambas, "A novel 4-D hyperchaotic Rikitake dynamo system with hidden attractor, its properties, synchronization and circuit design," Studies in Systems, Decision and Control, vol. 133, pp. 345-364, 2018.

[23] L. M. Pecora and T. L, Carroll, "Synchronization in chaotic systems," Phys. Rev. Lett., vol. 64, pp. 821-824, 1990.

[24] T. Yang, "A survey of chaotic secure communication systems," Internat. J. Computational Cognition, vol. 2, no. 2, pp. 81-130, 2004.

[25] H. Arabyani, N. Saberi, and Hassan, "Synchronisation of the hyperchaotic complex Lorenz system in a finite time," International Journal of Modelling, Identification and Control, vol. 25, no. 138, 2016, doi: 10.1504/IJMIC.2016.075273.

[26] A. Wolf, J. Swift, H. Swinney, and J. Vastano, "Determing Lyapunov exponents from a time series," Phys Lett B, vol. 737, no. 16D, pp. 285-317, 1985.

[27] P. Frederickson, J. L. Kaplan, E. D. Yorke, and J. A. Yorke, "The Liapunov dimension of strange attractors," J. Differ. Equ., vol. 49, no. 2. pp. 185-207, 1983. 